\title{
Communications
}

\section{Diffraction by a Dielectric Half-Plane}

\section{A. CHAKRABARTI}

\begin{abstract}
The problem of diffraction of a plane electromagnetic wave by a dielectric balf-plane is reconsidered for solution by ntilizing a set of recently developed approximate boundary conditions and the WienerHopf technique. It is observed from the present solution of the problem that reflected waves cease to exist if the angle of incidence takes up the value $\tan ^{-1}(1 / n)$ where $n$ represents the refractive index of the material of the half plane under consideration. Expression for the diffracted far field is obtained by using a modification of the saddle point method, and numerical values of the diffraction coefficients are presented in the form of a table for a special value of the refractive index $\boldsymbol{n}$ and for different values of the parameter $k h, h$ representing the (small) thickness of the half-plane and $k$ the wavenumber.
\end{abstract}

\section{INTRODUCTION}

Scattering by dielectric objects has several important practical applications as has been explained by Rawlins [1] where a set of approximate boundary conditions of an absorption type has been derived and utilized to study the problem of diffraction by an acoustically penetrable or an electrically dielectric half-plane. Similar diffraction problems have also been considered by Khrebet [7], Anderson [8], and Burnside and Burgener [9] by utilizing boundary conditions of different forms. Recently, Leppington [2] has derived a new set of approximate boundary conditions at the surfaces of a dielectric slab of small thickness surrounded by a different dielectric medium. Leppington's boundary conditions differ from the ones used in the works of [1], [7], [8], and [9] in the sense that they contain second order detivatives of the unknown potential function which are absent in an absorption type of boundary condition. With these new boundary conditions of Leppington we have reconsidered in the present communication the problem of scattering of an electromagnetic wave by a dielectric half-plane and, for the purpose of simplicity, we have restricted our attention only to the case of an incident plane wave. The problem is solved by using the WienerHopf technique, and Jones's method ([3], [4]) in a straightforward manner.

In Section I we formulate the problem and in Section II we demonstrate the Wiener-Hopf procedure for its solution. Detailed calculations are carried out for the determination of the reflected wave and the diffracted far field is expressed in terms of the Fresnel functions, by utilizing a modified saddle point method (see [5] and [6]). It turns out that the presently determined reflection coefficient is different from that obtained in [1], and this difference can be attributed to our modeling the problem through a different set of boundary conditions on the scatterer. Numerical values of the diffraction coefficient (see [8]) are tabulated for one particular choice of the ratio $\epsilon=\epsilon_{2} / \epsilon_{1}(=0.17)$ (mica or porcelain, see [5]) of the dielectric constants of the surrounding medium and the half-plane under consideration, respectively.

Manuscript received April 17, 1985; revised August 15, 1985.

The author is with the Department of Applied Mathematics, Indian Institute of Science, Bangalore 560 012, India.

IEEE Log Number 8608075.

\section{STATEMENT OF THE PRoblem}

A dielectric half-plane of dielectric constant $\epsilon_{1}$ and permeability $\mu$ occupies the region $-\infty<x<0, y=0$ of the $x-y$ plane $(z$-axis along the edge) which is supposed to be another dielectric medium of dielectric constant $\epsilon_{2}$ and the same permeability $\mu$ (for simplicity). A time-harmonic plane electromagnetic wave of potential $\phi_{i}=\exp \{-$ $\left.i k\left(x \cos \theta_{0}+y \sin \theta_{0}\right)-i \omega t\right\}$ falls on the half-plane $x<0$, with the understanding that the incident electromagnetic fields are given by

$$
\begin{aligned}
& \vec{E}_{i}=\operatorname{Re} \text { curl }\left(0,0, \phi_{i}\right) e^{-i \omega t} \\
& \vec{H}_{i}=-\operatorname{Re} i \omega \epsilon_{2}\left(0,0, \phi_{i}\right) e^{-i \omega t} .
\end{aligned}
$$

It is required to determine the scattered potential $\psi$, where $\psi=\phi-$ $\phi_{i}, \phi$ denoting the total potential, under a set of boundary conditions on the two surfaces of the half-plane to be described shortly and appropriate edge conditions and the radiation condition of outgoing waves at infinity. In what follows, we shall drop the time-dependent factor $e^{-i \omega t}$ and the symbol Re throughout. Then utility of Maxwell's equations shows that the governing partial differential equation for the function $\phi$ is

$$
\left(\nabla^{2}+k^{2}\right) \phi=0,
$$

where $k^{2}=\omega^{2} \mu \epsilon_{2}$, and, if we employ the boundary conditions derived by Leppington, we have that on $y=0$, for $x<0$,

$$
[\phi]=h\left(\frac{1}{\epsilon}-1\right)\left(\phi_{y}^{+}+\phi_{y}^{-}\right) \text {, }
$$

and

$$
\left[\phi_{y}\right]=h(1-\epsilon)\left(\phi_{x x}^{+}+\phi_{x x}^{-}\right),
$$

where the suffixes represent partial derivatives, $\epsilon=\epsilon_{2} / \epsilon_{1}, h(\ll 1)$ being the very small thickness of the half-plane under consideration. The symbol $[\phi]$ represents the jump $\left(\phi^{+}-\phi^{-}\right)$where $\phi^{+}$and $\phi^{-}$ are the limiting values of the function $\phi(x, y)$ as $y$ approaches zero from above and from below, respectively.

In order to obtain a unique solution it is necessary to specify the behavior of $\phi$ at the edge $x=0$. We shall require that $\phi, \phi_{x}$, and $\phi_{x x}$ possess at most an integrable singularity at this point.

With these boundary and edge conditions and the radiation condition of outgoing waves at infinity we next demonstrate the Wiener-Hopf procedure of the solution of our problem.

\section{METHOD OF SOLUTION}

The partial differential equation and the boundary conditions for the scattered potential $\psi$ are given by

$$
\begin{gathered}
\left(\nabla^{2}+k^{2}\right) \psi=0 \\
\psi\left(x, 0^{+}\right)-\psi\left(x, 0^{-}\right)-h\left(\frac{1}{\epsilon}-1\right)\left[\psi_{y}\left(x, 0^{+}\right)+\psi_{y}\left(x, 0^{-}\right)\right] \\
=-2 i k h\left(\frac{1}{\epsilon}-1\right) \sin \theta_{0} e^{-i k x \cos \theta_{0}}, \quad(x<0)
\end{gathered}
$$


and

$$
\begin{array}{r}
\psi_{y}\left(x, 0^{+}\right)-\psi_{y}\left(x, 0^{-}\right)-h(1-\epsilon)\left[\psi_{x x}\left(x, 0^{+}\right)+\psi_{x x}\left(x, 0^{-}\right)\right] \\
=-2 k^{2} h(1-\epsilon) \cos ^{2} \theta_{0} e^{-i k x \cos \theta_{0},} \quad(x<0) .
\end{array}
$$

Also, the conditions of continuity of $\psi$ and $\psi_{y}$ on $y=0$ for $x>0$ give that

$$
\psi\left(x, 0^{+}\right)=\psi\left(x, 0^{-}\right) \text {and } \psi_{y}\left(x, 0^{+}\right)=\psi_{y}\left(x, 0^{-}\right),(x>0) .
$$

We now use Fourier transforms of $\psi$ as defined by

$$
\Psi(s, y)=\int_{-\infty}^{\infty} \psi(x, y) e^{i s x} d x
$$

and

$$
\begin{aligned}
\Psi_{+}(s, y) & =\int_{0}^{\infty} \psi(x, y) e^{i s x} d x, \Psi_{-}(s, y) \\
& =\int_{-\infty}^{0} \psi(x, y) e^{i s x} d x
\end{aligned}
$$

and for the purpose of being able to utilize such transforms in our problem we imagine that $k$ has a positive imaginary part $k^{\prime}(k=k$ $\left.+i k^{\prime}\right)\left(k^{\prime}\right.$ will be taken to be zero at the end), so that the transform $\Psi$ exists and is analytic in the strip $-k^{\prime}<\operatorname{Im} s<k^{\prime} \cos \theta_{0}$ of the complex $s$-plane, while the transforms $\Psi_{+}$and $\Psi_{-}$are analytic in the overlapping half-planes $\operatorname{Im} s>-k^{\prime}$ and $\operatorname{Im} s<k^{\prime} \cos \theta_{0}$, respectively. Taking the Fourier transform of (4) and solving the resulting ordinary differential equation we find that

$$
\begin{aligned}
\Psi(s, y) & =A e^{-\gamma y}, y>0 \\
& =B e^{\gamma y}, y<0
\end{aligned}
$$

with $\gamma=\left(s^{2}-k^{2}\right)^{1 / 2}$, where that branch of the square root is understood for which $\gamma=-i k$ for $s=0$.

Applications of the Fourier transform to (5), (6), and (7), along with (9) result into the following relations:

$$
\left[\Psi^{\prime}\left(s, 0^{ \pm}\right)=\frac{d \Psi}{d y} \mid y=0^{ \pm}\right]:
$$

$$
\begin{gathered}
\Psi\left(s, 0^{+}\right)-\Psi\left(s, 0^{-}\right)-h\left(\frac{1}{\epsilon}-1\right)\left[\Psi^{\prime}\left(s, 0^{+}\right)+\Psi^{\prime}\left(s, 0^{-}\right)\right] \\
+P_{+}(s)=-\frac{2 k h\left(\frac{1}{\epsilon}-1\right) \sin \theta_{0}}{s-k \cos \theta_{0}},
\end{gathered}
$$

$\Psi^{\prime}\left(s, 0^{+}\right)-\Psi^{\prime}\left(s, 0^{-}\right)+h(1-\epsilon)\left[s^{2} \Psi\left(s, 0^{+}\right)+s^{2} \Psi\left(s, 0^{-}\right)\right]$

$$
+Q_{+}(s)=\frac{2 i k^{2} h(1-\epsilon) \cos ^{2} \theta_{0}}{s-k \cos \theta_{0}},
$$

$A-B=\Psi\left(s, 0^{+}\right)-\Psi\left(s, 0^{-}\right)=\Psi_{-}\left(s, 0^{+}\right)-\Psi_{-}\left(s, 0^{-}\right)=F_{-}(s)$

and

$$
\begin{aligned}
-\gamma(A+B) & =\Psi^{\prime}\left(s, 0^{+}\right)-\Psi^{\prime}\left(s, 0^{-}\right) \\
& =\Psi_{-}^{\prime}\left(s, 0^{+}\right)-\Psi_{-}^{\prime}\left(s, 0^{-}\right) \\
& =G_{-}(s)
\end{aligned}
$$

where

$$
P_{+}(s)=2 h\left(\frac{1}{\epsilon}-1\right) \int_{0}^{\infty} \psi_{y}(x, 0) e^{i s x} d x
$$

and

$$
Q_{+}(s)=2 h(1-\epsilon) \int_{0}^{\infty} \psi_{x x}(x, 0) e^{i s x} d x
$$

Using (9) in (10) and (11) and eliminating $A+B$ and $A-B$ with the help of (12) and (13) we arrive at the following two independent Wiener-Hopf equations for the unknowns $P_{+}, Q_{+}, F_{-}$and $G_{-}$:

$$
\left[1-h\left(\frac{1}{\epsilon}-1\right) \gamma\right] F_{-}+P_{+}=-\frac{2 k h\left(\frac{1}{\epsilon}-1\right) \sin \theta_{0}}{s-k \cos \theta_{0}}
$$

and

$$
\left[1-\frac{h(1-\epsilon) s^{2}}{\gamma}\right] G_{-}+Q_{+}=+\frac{2 i k^{2} h(1-\epsilon) \cos ^{2} \theta_{0}}{s-k \cos \theta_{0}} .
$$

The principal unknowns $A$ and $B$ are given by (using (12) and (13)):

$$
A=\frac{1}{2}\left[F_{-}-\frac{1}{\gamma} G_{-}\right] \text {and } B=\frac{-1}{2}\left[F_{-}+\frac{1}{\gamma} G_{-}\right] \text {. }
$$

The solution of the Wiener-Hopf equations (15) and (16) thus determine the transformed scattered potential in the two regions $y>$ 0 and $y<0$, with the help of (9), from which the potential $\psi(x, y)$ can be recovered by using Fourier inversion formula.

Using the factorization of the functions $f(s)$ and $g(s)$ as given by

$$
f(s)=1+h\left(\frac{1}{\epsilon}-1\right) \gamma=f_{+}(s) f_{-}(s)
$$

and

$$
g(s)=1-\frac{h(1-\epsilon) s^{2}}{\gamma}=g_{\div}(s) g_{-}(s)
$$

to be obtained by standard procedure (see Noble [3] and Leppington [2]) with $f_{ \pm} \sim 0\left(s^{1 / 2}\right)$, and $g_{ \pm} \sim 0\left(s^{1 / 2}\right)$ as $|s| \rightarrow \infty$ and free from zeros in corresponding half-planes, we arrive at the following solution of the Wiener-Hopf equations (15) and (16) with the help of Liouville's theorem:

$$
F_{-}(s)=-\frac{2 k h\left(\frac{1}{\epsilon}-1\right) \sin \theta_{0}}{f_{-}(s) f_{+}\left(k \cos \theta_{0}\right)\left(s-k \cos \theta_{0}\right)}
$$

and

$$
G_{-}(s)=+\frac{2 i k^{2} h(1-\epsilon) \cos ^{2} \theta_{0}}{g_{-}(s) g_{+}\left(k \cos \theta_{0}\right)\left(s-k \cos \theta_{0}\right)} .
$$

Then using (19), (20), and (17) in (9) and the Fourier inversion formula, we arrive at the result

$$
\psi(x, y)=\frac{k h(1-\epsilon)}{2 \pi i} \int_{-\infty}^{\infty} \frac{e^{-\gamma|y|-i s x}}{\left(s-k \cos \theta_{0}\right) \gamma}\left[\frac{k \cos ^{2} \theta_{0}}{g_{-}(s) g_{+}\left(k \cos \theta_{0}\right)}\right.
$$

$$
\left.-\frac{i\left(\frac{\sin \theta_{0}}{\epsilon}\right) \gamma \operatorname{sgn}(y)}{f_{-}(s) f_{+}\left(k \cos \theta_{0}\right)}\right] d s .
$$


The form (21) immediately gives that for $x>0$,

$$
\psi(x, 0)=\frac{k h(1-\epsilon)}{2 \pi i} \int_{-\infty}^{\infty} \frac{e^{i s x}}{\gamma\left(s-k \cos \theta_{0}\right)} \frac{k \cos ^{2} \theta_{0}}{g_{-}(s) g_{+}\left(k \cos \theta_{0}\right)} d s,
$$

obtained by deforming the contour for the other terms in the lower half-plane. Then, by Abelian theorems on Fourier transforms, we find that as $x \rightarrow 0^{+}$,

$$
\psi(x, 0) \sim 0\left(x^{3 / 2}\right) \text { and } \psi_{x x}(x, 0) \sim 0\left(x^{-1 / 2}\right) .
$$

We also obtain, in a similar fashion, from (14), that as $x \rightarrow 0^{+}$,

$$
\psi_{y}(x, 0) \sim 0\left(x^{-1 / 2}\right) .
$$

This is the actual edge behavior of the scattered field which was assumed in a different form beforehand in order to apply the WienerHopf procedure to our problem.

For a full knowledge of the scattered field $\psi(x, y)$ we observe that details of the split functions $f_{ \pm}$and $g_{ \pm}$are very much required, while if our interest is just to calculate the reflection coefficient this can be avoided. We find that for $x<0$, the reflected wave as given by

$$
\mathrm{RF}=\operatorname{Re}^{-i k\left(x \cos \theta_{0}-y \sin \theta_{0}\right)}
$$

can be calculated from (21) by deforming the contour in the upper half-plane, when the pole $s=k \cos \theta_{0}$ is captured and we obtain the reflection coefficient $R$ as given by with

$$
Q=\left(\frac{k r}{2}\right)^{1 / 2}\left(\frac{\cos \theta+\cos \theta_{0}}{\sin \theta}\right)
$$

and

$$
\hat{F}(Z)=e^{i z^{2}} \int_{z}^{\infty} e^{i t^{2}} d t
$$

In order to utilize (25) for computational purposes, we shall obtain approximate expressions for the factors $f_{ \pm}$and $g_{ \pm}$by following Leppington's analysis [2].

Writing

$$
g(s)=\left(s^{2}-k^{2}\right)^{-1 / 2} g *(s),
$$

and

$$
f(s)=\frac{\left(s^{2}-k^{2}\right)^{1 / 2}}{\epsilon} f *(s)
$$

with

and

$$
\left.\begin{array}{l}
g^{*}(s)=\left(s^{2}-k^{2}\right)^{1 / 2}-\epsilon_{0} s^{2} \\
f *(s)=\epsilon\left(s^{2}-k^{2}\right)^{-1 / 2}+\epsilon_{0}
\end{array}\right\}\left[\epsilon_{0}=h(1-\epsilon)\right]
$$

$$
R=\frac{i k h(1-\epsilon)\left(\cos ^{2} \theta_{0}-\sin ^{2} \theta_{0} / \epsilon\right)}{1-i k h(1-\epsilon)\left(\sin ^{2} \theta_{0}+\epsilon \cos ^{2} \theta_{0}\right) /\left(\epsilon \sin \theta_{0}\right)-k^{2} h^{2}(1-\epsilon)^{2} \cos ^{2} \theta_{0} / \epsilon} .
$$

The transmission coefficient also can be completely calculated in a similar fashion. We observe that $R=0$ is possible if $\theta_{0}=\tan ^{-1}$ $\left(\epsilon^{1 / 2}\right)=\tan ^{-1}\left(k / k_{1}\right)=\tan ^{-1}(1 / n)$, with $k_{1}=n k, n$ being the refractive index of the half-plane under consideration.

In order to determine the diffracted field we proceed as follows.

Changing $s$ to $-s$ in (21) we express $\psi(x, y)$ in the form

$$
\psi(x, y)=\frac{1}{2 \pi i} \int_{-\infty}^{\infty} \frac{H(s)}{\left(s-s_{p}\right)\left(k^{2}-s^{2}\right)^{1 / 2}} e^{i\left[s x+\left(k^{2}-s^{2}\right)^{1 / 2}|y|\right]} \mathrm{ds},
$$

where $s_{p}=-k \cos \theta_{0}$, and

$$
\begin{aligned}
H(s)=\frac{k h(1-\epsilon)}{i}\left[\frac{k \cos ^{2} \theta_{0}}{\frac{g_{+}(s) g_{+}\left(k \cos \theta_{0}\right)}{\left(\frac{\sin \theta_{0}}{\epsilon}\right)\left(k^{2}-s^{2}\right)^{1 / 2} \operatorname{sgn}(y)}}\right] \\
\left.-\frac{(s) f_{+}\left(k \cos \theta_{0}\right)}{f_{+}(s)}\right] .
\end{aligned}
$$

Then following Rawlins [6], after substituting $x=r \cos \theta, y=r$ $\sin \theta,(-\pi<\theta<\pi)$, we observe that the diffracted far field $\psi_{\text {diff }}(r$, $\theta)$, valid for large $k r$, can be represented in the form

$$
\psi_{\text {diff }}\left\{\begin{array}{cc}
D_{+}, & \text {for } 0<\theta<\pi \\
D_{-}, & \text {for }-\pi<\theta<0
\end{array}\right.
$$

where

$$
\begin{aligned}
D_{ \pm}= & h(1-\epsilon) \frac{e^{i(k r+\pi / 4)}}{(2 \pi k r)^{1 / 2}} \frac{2|Q| \hat{F}(|Q|)}{\left(\cos \theta+\cos \theta_{0}\right)} \\
& .\left[\frac{k \cos ^{2} \theta_{0}}{g_{+}(k \cos \theta) g_{+}\left(k \cos \theta_{0}\right)}\right. \\
& \left.\mp \frac{k \sin \theta \sin \theta_{0} / \epsilon}{f_{+}(k \cos \theta) f_{+}\left(k \cos \theta_{0}\right)}\right],
\end{aligned}
$$

we find that as $h \rightarrow 0$,

$$
\begin{aligned}
g_{+}(s) \sim 1-\frac{2 \epsilon_{0} s^{2}}{\left(s^{2}-k^{2}\right)^{1 / 2}} \tan ^{-1}\left(\frac{k-s}{k+s}\right)^{1 / 2} \\
+s\left[\left(\frac{1}{2}+\frac{i}{\pi}\right) \epsilon_{0}-\frac{i \epsilon_{0}}{\pi} \ln \left(\frac{\epsilon_{0} k}{2}\right)\right]
\end{aligned}
$$

and

$$
\begin{aligned}
& f_{+}(s) \sim 1+\frac{\epsilon_{0}}{\epsilon} {\left[\frac{2}{\pi}\left(s^{2}-k^{2}\right)^{1 / 2} \tan ^{-1}\left(\frac{k-s}{k+s}\right)^{1 / 2}\right.} \\
&\left.+\left(\frac{1}{2}-\frac{i}{\pi}\right) s+\frac{i s}{\pi} \ln \left(\frac{\epsilon_{0} k}{2}\right)\right] .
\end{aligned}
$$

We must note that these forms of the factors are nonuniform when either $s$ is close to $\pm k$ or when $|s|$ is large. But for the purpose of computing the diffracted far field with the help of (25), the expressions (27) are useful except when $s$ is near $\pm k$. When $s$ is near $\pm k$, we again adopt Leppington's analysis and obtain the following results.

Near $s=k$,

$$
g_{+}(s) \sim 1, g_{-}(s) \sim(s-k)^{-1 / 2}\left[(s-k)^{1 / 2}-2^{-1 / 2} \epsilon_{0} k^{3 / 2}\right]
$$

and

$$
f_{+}(s) \sim 1, f_{-}(s) \sim 1+\frac{\epsilon_{0}}{\epsilon}(2 k)^{1 / 2}(s-k)^{1 / 2},
$$

and near $s=-k$ (change $s$ to $-s$ in above and use $(s-k)^{1 / 2}=-i$ $\left.(k-s)^{1 / 2}\right)$

$$
\left.g_{-}(s)-1, g_{+}(s) \sim(s+k)^{-1 / 2}[s+k)^{1 / 2}-e^{i \pi / 2} 2^{-1 / 2} \epsilon_{0} k^{3 / 2}\right]
$$

and

$$
f_{-}(s) \sim 1, f_{+}(s) \sim 1+\frac{\epsilon_{0}}{\epsilon} e^{-i \pi / 2}(2 k)^{1 / 2}(s+k)^{1 / 2} .
$$

The results (27), (28), and (29) help the computation of the diffracted 
TABLE I

DiFFRACTION COEFFICIENTS $C_{\text {diff }}$ FOR NORMAL INCIDENCE $\left(\theta_{0}=90^{\circ}\right)$

\begin{tabular}{|c|c|c|c|}
\hline$\theta$ & $c_{\text {diff }}(k h=0.001)$ & $c_{\text {diff }}(\mathrm{kh}=0.01)$ & $c_{\text {alff }}(x h=0.1)$ \\
\hline $0^{\circ}$ & 0 & 0 & 0 \\
\hline $10^{\circ}$ & 0.00085666 & 0.00818086 & 0.05412580 \\
\hline $20^{\circ}$ & 0.00176842 & 0.01690647 & 0.11283916 \\
\hline $30^{\circ}$ & 0.00280503 & 0.02686441 & 0.18190884 \\
\hline $40^{\circ}$ & 0.00407386 & 0.03910989 & 0.27014369 \\
\hline $50^{\circ}$ & 0.00576719 & 0.05552835 & 0.39323780 \\
\hline $60^{\circ}$ & 0.00827117 & 0.07990407 & 0.58279179 \\
\hline $70^{\circ}$ & 0.01241746 & 0.12039714 & 0.90797999 \\
\hline $80^{\circ}$ & 0.01998179 & 0.19447967 & 1.52062260 \\
\hline $90^{\circ}$ & 0.03421254 & 0.33427254 & 2.71495580 \\
\hline $100^{\circ}$ & 0.01999867 & 0.19613622 & 1.65556630 \\
\hline $110^{\circ}$ & 0.01243818 & 0.12242526 & 1.07294300 \\
\hline $120^{\circ}$ & 0.00829137 & 0.08187863 & 0.74328041 \\
\hline $130^{\circ}$ & 0.00578531 & 0.05729756 & 0.53675038 \\
\hline $140^{\circ}$ & 0.00408911 & 0.04059845 & 0.39057056 \\
\hline $150^{\circ}$ & 0.00281691 & 0.02802241 & 0.27530459 \\
\hline $160^{\circ}$ & 0.00177655 & 0.01769817 & 0.17649384 \\
\hline $170^{\circ}$ & 0.00086078 & 0.00858258 & 0.08634135 \\
\hline $180^{\circ}$ & 0 & 0 & 0 \\
\hline
\end{tabular}

fields $D_{ \pm}$by means of the formulas (25) and (26). We have computed the numerical values of the diffraction coefficient (see [8]) as defined by

$$
C_{\text {diff }}=\left|(2 \pi k r)^{1 / 2} \psi_{\text {diff }}\right|=\frac{\left(k \epsilon_{0} / \epsilon\right)(2 k r)^{1 / 2}|\hat{F}(|Q|)|}{\left|f_{\dot{*}}(0)\right|\left|f_{+}(k \cos \theta)\right|}
$$

for the case of normal incidence $\left(\theta_{0}=90^{\circ}\right)$ and for different values of $k h(=0.001,0.01$, and 0.1$)$ with $k r=10 \pi$, for a special value of $\epsilon(=0.17)$, representative of mica or porcelain materials (cf. [5]). These values are presented in the form of a table, instead of graphs, in order to see the variations clearly.

\section{ACKNOWLEDGMENT}

I am grateful to the referee for bringing the three papers in [7], [8], and [9] to my notice and for several important suggestions made to improve the presentation of the article.

\section{REFERENCES}

[1] A. D. Rawlins, "Diffraction by an acoustically penetrable or an electromagnetically dielectric half-plane," Int. J. Eng. Sci., vol. 15, pp. 569-578, 1977.

[2] F. G. Leppington, "Travelling waves in a dielectric slab with an abrupt change in thickness," Proc. Roy. Soc. Lond. A, vol. 386, pp. 443$460,1983$.

[3] B. Noble, Methods Based on the Wiener-Hopf Technique. Oxford: Pergamon, 1958.

[4] A. Chakrabarti, "A note on Jones's method associated with the Wiener-Hopf technique," Int. J. Math. Educ. Sci. Technol., vol. 12, pp. 597-602, 1981.

[5] D. S. Jones, The Theory of Electromagnetism. Oxford: Pergamon,
1964, p. 689.

[6] A. D. Rawlins, "Acoustic diffraction by an absorbing semi-infinite half-plane in a moving fluid," Proc. Roy. Soc. Edin., vol. 72, pp. 337-357, 1974.

[7] N. G. Khrebet, "Diffraction of plane electromagnetic waves on the edge of a dielectric half-plane," Radio Eng. Electron. Phys., (USSR), vol. 13, pp. 331-338, 1968.

[8] I. Anderson, "Plane wave diffraction by a thin dielectric half-plane," IEEE Trans. Antennas Propagat., vol. AP-27, no. 5, pp. 584-589, 1979.

[9] W. D. Burnside and K. W. Burgener, "High frequency scattering by a thin lossless dielectric slab," IEEE Trans. Antennas Propagat., vol. AP-31, no. 1, pp. 104-110, 1983.

\section{Diffraction of a TM Whispering Gallery Mode by the Edge of a Nonperfectly Conducting Spherically Curved Sheet}

\section{A. HAMIT SERBEST, MEMBER, IEEE}

Abstract-Electromagnetic scattering is a function of the scatterer's material properties as well as its geometry. Therefore, it is necessary to

Manuscript received March 30, 1985; revised August 21, 1985.

The author is with the Faculty of Engineering, Çukurova University, Balcali, PK 198, Adana, Turkey.

IEEE Log Number 8608077. 